\title{
Virtual Avatars as a tool for audience engagement
}

\author{
Maria Zelenskaya \\ Griffith Film School \\ maria.zelenskaya@gmail.com
}

\author{
Louise Harvey \\ Griffith Film School \\ 1.harvey@griffith.edu.au
}

\begin{abstract}
Modern motion capture tools can be used to animate sophisticated digital characters in real time. Through these virtual avatars human performers can communicate with live audience, creating a promising new area of application for public engagement. This study describes a social experiment where a real-time multimedia setup was used to facilitate an interaction between a digital character and visitors at a public venue. The technical implementation featured some innovative elements, such as using iPhone TrueDepth Camera as part of the performance capture pipeline. The study examined public reactions during the experiment in order to explore the empathic potential of virtual avatars and assess their ability to engage live audience.
\end{abstract}

\section{CCS CONCEPTS}

Computing methodologies $\rightarrow$ Computer graphics $\rightarrow$ Animation $\rightarrow$ Motion capture.

\section{KEYWORDS}

Real-time motion capture, virtual avatar, audience engagement.

\section{ACM Reference format:}

Maria Zelenskaya and Louise Harvey. 2019. Virtual Avatars as a tool for audience engagement. In Proceedings of VRCAI'19: The 17th International Conference on Virtual-Reality Continuum and its Applications in Industry (VRCAI '19), November 14-16, 2019, Brisbane, QLD, Australia. ACM, New York, NY, USA, 3 pages. https://doi.org/10.1145/3359997.3365717

\section{INTRODUCTION}

Motion Capture (MoCap) is a process of tracking an actor's movements and converting them into a lifelike animation of a digital character [Delbridge, 2015]. Advanced MoCap systems are widely used in video production and can capture full body performance with great precision, including finger movements and facial expressions. Recent developments in real-time animation and rendering technology are creating opportunities for new interactive applications [Petit, 2017].
Virtual avatars - digital characters that are being controlled by a human actor, are the latest trend in the entertainment industry. With more affordable MoCap solutions on the market, virtual avatars are becoming very popular [Schindegger, 2019]. They are appearing at conferences, live TV shows and promotional events, making live character animation a promising new area of application for public engagement.

\section{PROBLEM AND APPROACH}

The general appeal of traditional animation is well known [Wasko, 2001], and the use of animated characters in advertising has been extensively studied [Huang et al., 2011]. However, live character animation, such as in the form of virtual avatars, is a new phenomenon and is under-explored in the academic literature.

To investigate the entertainment potential of virtual avatars and their ability to engage spectators and to invoke an emotional response, a social experiment was conducted to observe live interactions between a virtual character and the general public. The experiment was facilitated through a low-budget multimedia setup that applied the latest trends in real-time motion capture technology in order to allow a human performer to control a 3D character and communicate with live audience.

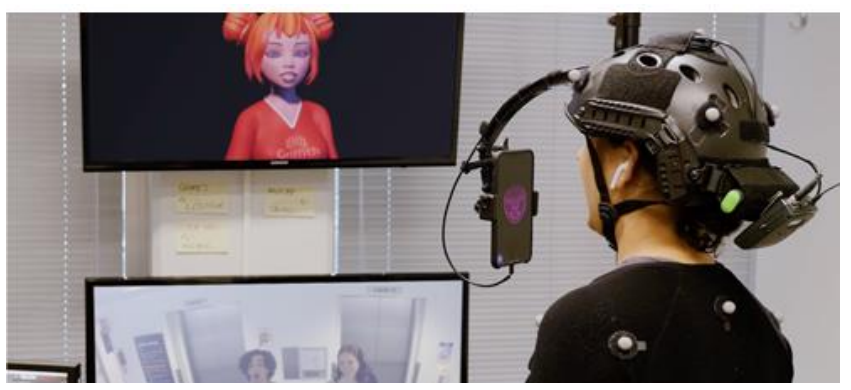

Figure 1: The performer controlling her 3D avatar, 2019

\section{EXPERIMENT}

In August 2019 Griffith University's Queensland College of Art hosted an Open Day event drawing a large number of visitors. At a designated location on campus visitors were greeted by a virtual character, Paige, from a large TV screen installed on a wall. Paige initiated conversations with the guests, engaging them with helpful advice about the college and directing them to various attractions. 
After a brief interaction, each group of visitors was invited to take a look behind the scenes and meet the human performer, situated in another room nearby (See Figure 1).

\subsection{Character creation}

Paige was designed as a simple friendly character with attractive anime style features to appeal to the young adult audience. The model was created using Daz Studio ${ }^{1}$, a 3D software with powerful character customisation tools. The skeletal mesh was imported into the 3D program Autodesk $\mathrm{Maya}^{2}$, where a Human IK skeletal rig was defined. Blend shapes were set up to provide 51 unique facial expressions, according to Apple's ARkit. Finally, Paige was imported into Unreal Engine ${ }^{3}$.

\subsection{Performance capture}

To track the performer's body movements, an optical motion capture system was used, comprising of 12 Vicon Vantage infrared cameras installed in a small room. The motion data was streamed live from Vicon's proprietary software Shogun into Autodesk MotionBuilder ${ }^{4}$ for retargeting. Retargeted animation was streamed from MotionBuilder to Unreal Engine through a Livelink plugin and applied to Paige. Facial expressions were captured using an iPhone, mounted to the performer's helmet. The motion data captured by iPhone's TrueDepth camera and Apple ARkit was streamed over Wi-Fi straight into Unreal Engine though the LiveLink Plugin (See Figure 2)

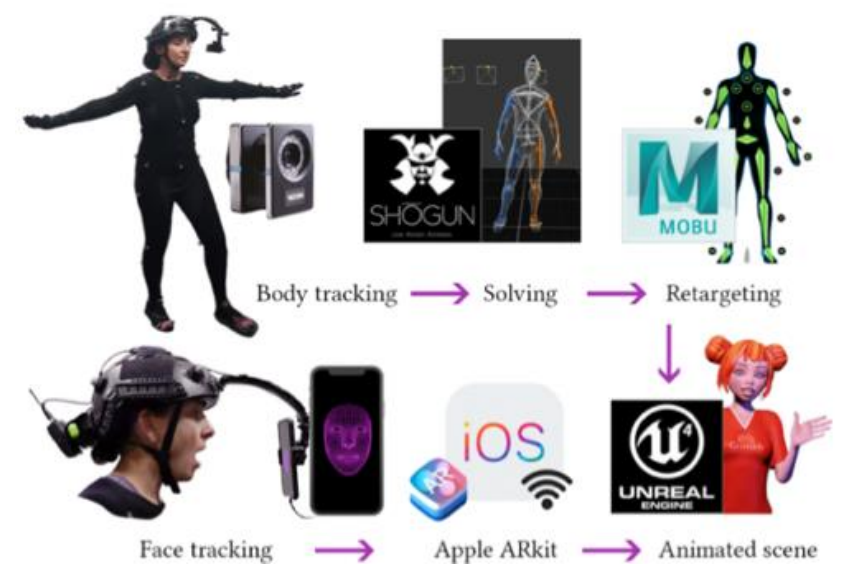

Figure 2: Animation pipeline, used during experiment

\subsection{Multimedia streaming}

The screen displaying the real-time scene was captured and streamed over the local area network onto a small computer, hidden behind the TV. The audio was captured by a wireless microphone attached to the performer's helmet. A microphone and a video camera were installed above the TV screen where Paige was visible to visitors, recording the audience. The video feed was

\footnotetext{
${ }^{1}$ https://www.daz3d.com

${ }^{2}$ https://www.autodesk.com.au/products/maya

${ }^{3}$ https://www.unrealengine.com

${ }^{4}$ https://www.autodesk.com/products/motionbuilder
}

displayed on a monitor in front of the performer. The audio was streamed to her Bluetooth earpiece.

No significant synchronisation issues were detected between the multiple media streams.

Conveying gaze direction during video conferencing presents a technical challenge, especially on a single 2D planar display [Pan et al., 2014]. We were able to avoid significant misalignment in the natural lines of sight by carefully matching the camera's vantage point with the position of the monitor in front of the performer.

\section{RESULTS AND EVALUATION}

Several hours of video footage were recorded during the experiment, capturing multiple short interactions between Paige and small groups of visitors. Each interaction was unscripted and spontaneous. From these interactions, several common trends were observed. Paige seemed to make a strong initial impression and was successful at drawing spectators closer to the screen. However, many people were hesitant to engage in a more meaningful conversation. Some appeared wary of cameras positioned in the area. Most guests were curious to meet the performer behind the scenes and chat with her. After meeting the performer, visitors were more likely to go back to the interactive screen and continue their conversation with Paige.

\section{CONCLUSION}

A virtual avatar was successfully used to engage a live audience during Griffith University Open Day. The novelty factor was the biggest driver for spectators, attracting them to the screen. Despite every effort for preserving immersion, the public was still aware of the cameras and other technicalities. Observational data indicates that some people prefer to establish a human connection first, before they can comfortably connect with a virtual character. The overall response, however, was very positive, signifying the entertainment potential of real-time virtual avatars.

\section{ACKNOWLEDGMENTS}

The authors would like to thank Narelle Vella for performing the role of Paige, James Stafford and Curtis Sullivan for technical support, Immerse Enterprise for consulting expertise on virtual avatars.

\section{REFERENCES}

Delbridge, Matt. Motion capture in performance: an introduction. Springer, 2015 Huang, Wen-Shin, Tsuifang Hsieh, and Han-Shen Chen. "The advertisement effectiveness of animated spokes-characters." African journal of business management 5, no. 23 (2011): 9971-9978.

Pan, Ye, William Steptoe, and Anthony Steed. "Comparing flat and spherical displays in a trust scenario in avatar-mediated interaction." In Proceedings of the 32 nd annual ACM conference on Human factors in computing systems, pp. 1397-1406. ACM, 2014.

Petit, Marc. "Realtime technology blurs the lines between production \& post." Post, July 2017, p. 36

Schindegger, Jacob, "The Future Group, Xsens and IKINEMA partner to bring plugand-play real-time character animation to the broadcast industry", The Future Group press release, Accessed 23 May 2019

Wasko, Janet. Understanding Disney: The Manufacture of Fantasy. Cambridge, UK;Oxford;Malden, MA;: Polity. 2001 